Pacific Journal of Mathematics

EXISTENCE AND REGULARITY FOR THE PROBLEM OF A

GLEL, UMberto Massari and I. TAMANINI 


\title{
EXISTENCE AND REGULARITY FOR THE PROBLEM OF A PENDENT LIQUID DROP
}

\author{
E. Gonzalez, U. MAssari AND I. TAMAnini
}

The subject of this paper is the study of the existence of a pendent drop. We carry out this study in full generality by exploiting the local minima of a suitable functional, chosen to represent the energy of the drop.

If we denote by $E \subset \boldsymbol{R}^{n+1}$ a liquid drop hanging from the fixed horizontal reference plane $\{t=0\}$, then we can write the global energy of that configuration in the following way:

$$
\mathscr{F}(E)=\int_{t<0}\left|D \varphi_{E}\right|+\nu \int_{t=0} \varphi_{E} d H_{n}+\kappa \int_{t<0} t \varphi_{E}(x, t) d x d t
$$

Here, the first integral is the measure of that part of the boundary of $E$ lying in the half-space $\{t<0\}$. Physically, it corresponds to the energy due to surface tension. The second integral, proportional to the measure of the boundary of $E$ contacting the horizontal plane, represents the energy given by the attraction between the liquid and the plane itself, while the third one corresponds to the gravitational energy.

Exact definitions of these objects will be given in the next section.

The constant $\nu$ is determined experimentally, depending on the materials in the liquid-solid-vapor interface; physically, it represents the cosine of the angle between the exterior directed normal to the liquid surface, along the intersection with the contact plane $\{t=0\}$, and the positive (vertical) $t$-direction. The constant $\kappa \geqq 0$ takes into account the gravitational acceleration, and is referred to as the "capillary constant" (see e.g., [12]).

We have to minimize the functional (0.1) among the sets of finite perimeter (in the sense of De Giorgi; see [7] or [11], where the equivalent notion of mass is used), having prescribed $V$ volume. It is clear that we cannot expect a finite lower bound for (0.1) in such a class, as physical considerations may suggest. Indeed, a pendent drop is just a local minimum of the energy functional.

In order to prove the existence of such a local minimum, we introduce a ground floor (i.e., a plane $\{t=T\}$ with a suitable $T<0$ ) and minimize the energy among those configurations $E$ lying between floor and ceiling: for small gravity, we can prove that such minima do not reach the floor. We do this by observing that as gravity 
decreases to zero, the corresponding minima approach - in the sense of a good convergence - the solution in the absence of gravity, which is part of a sphere.

We remark that when it is possible to describe the part of the boundary of $E$, lying below the contact plane, as the graph of a function $u \in C^{2}(\Omega), \Omega$ open in $\boldsymbol{R}^{n}$, then we can write the functional (0.1) in the equivalent way

$$
\mathscr{F}(u)=\int_{\Omega} \sqrt{1+|D u|^{2}}+\nu|\Omega|-\frac{\kappa}{2} \int_{\Omega} u^{2} d x
$$

It follows that a minimum of (0.2) satisfies the Euler equation

$$
\operatorname{div} T u=-\kappa u+\lambda, \quad T u=D u \cdot\left(1+|D u|^{2}\right)^{-1 / 2}
$$

in which $\lambda$ is a constant ensuing from the volume constraint.

In the physical case $n=2$, considering only rotationally symmetric solutions (i.e., solutions symmetric about the vertical $t$-axis), equation (0.3) takes the simpler form:

$$
\frac{1}{r}\left(\frac{r u^{\prime}(r)}{\sqrt{1+u^{\prime 2}(r)}}\right)^{\prime}=-\kappa u(r)+\lambda
$$

with $u=u(r)$.

The behavior of a solution of (0.4), in its dependence on the initial value $u(0)=u_{0}<0$, has been studied extensively by P. Concus and R. Finn in a series of papers $([12,2,3,4]$; see also $[28,29])$. We refer to [4] for a recent detailed exposition on this argument.

Several interesting results dealing with equation (0.4) have also been obtained from the computational point of view; see [12] and the references cited there for a general account.

The question of existence of a drop suspended from an arbitrary aperture, consisting of a simple closed curve in $\boldsymbol{R}^{3}$, was studied by H. C. Wente in [34]. The method employed in that paper still involves minimization of a suitable functional, however by means of complexvariable techniques, and gives an affirmative answer for sufficiently small gravitational field; it differs completely from our method, which relies on De Giorgi's Theory of Perimeter and works in any dimension.

In this framework, developed in [5], [6], [7] (see also [1], [19] for a detailed treatment of the subject), several capillarity problems have been recently solved; first of all, the problem of existence of equilibrium surfaces in a capillary tube, whose solution was obtained by M. Emmer in [9] (see also [13], [32] for extensions of Emmer's result to capillary tube of general cross-section, and [27], [14], [15], [30], [31], [33], [17] for interior analiticity and boundary regularity of Emmer's solution). E. Gonzalez and I. Tamanini ([20], [21], [22]) 
studied subsequently the problem of a sessile drop (i.e., a liquid drop sitting on a flat plate), proving existence, regularity and convexity of the equilibrium configuration. Further contributions along this line were obtained by U. Massari and L. Pepe in [25], [26].

Definitions and notations to be used in the sequel are given in the first section. Section 2 is devoted to the gravity-free problem, that is to the study of the minima of (0.1) with $\kappa=0$, while $\varepsilon$-gravity problems are studied in $\S 3$. Sections 4 and 5 deal with some properties of $\varepsilon$-solutions that are used in the last two sections to present the conclusive existence and regularity results.

For convenience of the reader, some questions related to the existence of multipliers have been quoted in the Appendix.

We wish to thank P. Concus for many helpful suggestions on the use of comparison surfaces, in $\S 6$.

When writing the manuscript we were informed by E. Giusti that he obtained an analogous existence result, without any use of comparison surfaces.

1. Notation and definitions. We denote by $z=(x, t)$, with $x \in \boldsymbol{R}^{n}$ and $t \in \boldsymbol{R}(n \geqq 2)$, an arbitrary point in $\boldsymbol{R}^{n+1}$, by $H_{s}$ the $s$ dimensional Hausdorff measure ([11]), by $B V(\Omega)$ the set of Lebesgue integrable functions $f(y)$ over the open subset $\Omega$ of $\boldsymbol{R}^{m}$, whose gradient, in the sense of distributions, is a vector measure with finite total variation. That is,

$$
B V(\Omega)=\left\{f \in L^{1}(\Omega): \int_{\Omega}|D f|<+\infty\right\}
$$

where

$$
\int_{\Omega}|D f|=\sup \left\{\int_{\Omega} f(y) \operatorname{div} g(y) d y: g \in\left[C_{0}^{1}(\Omega)\right]^{m},|g| \leqq 1\right\} .
$$

We refer to the quantity $\int_{\Omega}\left|D \varphi_{E}\right|$, involving the characteristic function $\varphi_{E}$ of a Borel set $E \subset \boldsymbol{R}^{m}$, as the perimeter of $E$ in $\Omega$; when $\Omega=\boldsymbol{R}^{m}$, we simply write $\int\left|D \varphi_{E}\right|$, the perimeter of $E$.

If the boundary $\partial \Omega$ of $\Omega$ is locally Lipschitz, then ([23]) each function $f \in B V(\Omega)$ has a trace belonging to $L^{1}(\partial \Omega)$. The functional (0.1) is therefore well-defined on sets $E$ having finite perimeter in $\boldsymbol{R}^{n+1}$; of course, $\int_{t<0}\left|D \varphi_{E}\right|$ means the perimeter of $E$ in the half-space $\Omega=\{(x, t): t<0\}$, while $\int_{t=0} \varphi_{E} d H_{n}$ corresponds to the trace over $\partial \Omega$. Setting for $T<0$

$$
S_{T}=\{(x, t): T<t<0\},
$$


by $A \subseteq S_{T}$ we intend that there exists $\delta>0$ such that $A \subset S_{T++j}$. We call $G$ (a set of finite perimeter in $\boldsymbol{R}^{n+1}$ ) a local minimum for the functional $\mathscr{F}$ defined by $(0.1)$ - a pendent $d r o p$ - if there exists $T<0$ such that

(i) $G \Subset S_{T}$

(ii) for each $F$ of finite perimeter, with $|F|=|G|$ and $F \triangle$ $G \in S_{T}$, the inequality $\mathscr{F}(G) \leqq \mathscr{F}(F)$ holds, where:

$$
F \triangle G=(F-G) \cup(G-F) \text { and }|F|=H_{n+1}(F) .
$$

2. Free-gravity problem. Consider the functional

$$
\mathscr{F}_{0}(E)=\int_{t<0}\left|D \mathcal{P}_{E}\right|+\nu \int_{t=0} \mathcal{P}_{E} d H_{n}
$$

in the class

$$
\mathscr{E}=\left\{E \subset\{t<0\}: \int\left|D \phi_{E}\right|<+\infty,|E|=V\right\}
$$

where $V>0$ is a fixed constant and $\nu \in \boldsymbol{R}$.

When $|\nu|<1$, the isoperimetric inequality ([8], [18]) implies at once the existence of a unique minimum for $\mathscr{F}_{0}$ in $\mathscr{E}$. Such a minimum $E_{0}$ is the intersection of the half-space $\{t<0\}$ with a ball, centered at the point $c_{0}$ on the $t$-axis and having radius $R_{0}$; radius and position of the center are to be determined in such a way that the measure of the intersection is $V$ and the cosine of the contact angle is $\nu$. That is

$$
E_{0}=\left\{(x, t) \in \boldsymbol{R}^{n+1}: t<0,|x|<\rho_{0}(t)=\left(R_{0}^{2}-\left(t-c_{0}\right)^{2}\right)^{1 / 2}\right\}
$$

where $R_{0}, c_{0}$ are to be determined through the relations

$$
\left|E_{0}\right|=V ; \quad \nu=-\frac{\rho_{0}^{\prime}(0)}{\sqrt{1+\rho_{0}^{\prime 2}(0)}} .
$$

The minimum height of the solution $E_{0}$ is given by

$$
Q_{0}=c_{0}-R_{0}=\frac{-(1+\nu) V^{1 / n+1}}{\left(\omega_{n} \int_{-1}^{\nu}\left(1-s^{2}\right)^{n / 2} d s\right)^{1 / n+1}} .
$$

We remark moreover that every ball of volume $V$, lying in $\{t<0\}$, is a solution of the problem in the case $\nu=1$, while for $\nu=-1$ no solution can occur.

3. E-gravity problems. We prove in this section that the functional 


$$
\mathscr{F}_{\varepsilon}(E)=\int_{S_{T}}\left|D \varphi_{E}\right|+\nu \int_{t=0} \varphi_{E} d H_{n}+\int_{t=T} \varphi_{E} d H_{n}+\varepsilon \int_{S_{T}} t \varphi_{E}(z) d z
$$

attains its minimum in the class

$$
\mathscr{E}_{T}=\left\{E \subset S_{T}: \int\left|D \varphi_{E}\right|<+\infty,|E|=V\right\}
$$

for every $\varepsilon>0$.

With respect to the minimization of $\mathscr{F}_{\varepsilon}$, we may restrict ourselves to rotationally symmetric sets. Indeed, if for $E \in \mathscr{E}_{T}$ we define

$$
\begin{aligned}
\rho(t) & =\left(\omega_{n}^{-1} \int \varphi_{E}(x: t) d x\right)^{1 / n} \\
E^{s} & =\left\{(x, t) \in \boldsymbol{R}^{n+1},|x|<\rho(t)\right\}
\end{aligned}
$$

then we derive from Lemma 2 in [20]:

$$
\mathscr{F}_{\varepsilon}\left(E^{s}\right) \leqq \mathscr{F}_{\varepsilon}(E)
$$

equality holding if and only if $E^{s}=E$, that is, the set $E$ is already symmetric. We define

$$
\tilde{\mathscr{E}}_{T}=\left\{E^{s}: E \in \mathscr{E}_{T}\right\}
$$

Lemma 3.1. For $|\nu| \leqq 1$, the functional $\mathscr{F}_{\mathrm{s}}$ defined by (3.1) has a finite lower bound on $\mathscr{E}_{T}$.

Proof. Clearly we have

$$
\int_{t<0}\left|D \varphi_{E}\right|>\int_{t=0} \varphi_{E} d H_{n}
$$

for any $E \in \mathscr{E}_{T}$, from which we obtain

$$
\begin{aligned}
0 & \leqq \frac{1-\nu}{2} \int_{t<0}\left|D \varphi_{E}\right|+\frac{\nu-1}{2} \int_{t=0} \varphi_{E} d H_{n} \\
& =\left(1-\frac{1+\nu}{2}\right) \int_{t<0}\left|D \varphi_{E}\right|+\left(\nu-\frac{1+\nu}{2}\right) \int_{t=0} \varphi_{E} d H_{n}
\end{aligned}
$$

and then

$$
\mathscr{F}_{\varepsilon}(E)>\frac{1+\nu}{2} \int\left|D \varphi_{E}\right|+\varepsilon T V
$$

which concludes the proof.

LEMMA 3.2. Let $\left\{E_{h}\right\}$ be a sequence of sets in $\tilde{\mathscr{E}}_{T}$ such that $\mathscr{F}_{\varepsilon}\left(E_{h}\right)<$ const. $\forall$. If $-1<\nu \leqq 1$, then a subsequence of $\left\{E_{h}\right\}$ converges in $L^{1}\left(\boldsymbol{R}^{n+1}\right)$ to a limit set $E \in \tilde{\mathscr{E}}_{T}$. 
Proof. The existence of a subsequence of $\left\{E_{h}\right\}$, convergent in $L_{\text {loc }}^{1}\left(\boldsymbol{R}^{n+1}\right)$, follows easily from (3.4) together with a known compactness result ([24]). Now, we have for almost all $t<0$ :

$$
\int \varphi_{E_{h}}(x, t) d x \leqq \int\left|D \varphi_{E_{h}}\right| \leqq \text { const } .
$$

We can thus find a ball $B_{R} \subset \boldsymbol{R}^{n}$, such that every set in the sequence lies in the cylinder $B_{R} \times(T, 0)$. Hence, convergence of the previous subsequence actually takes place in $L^{1}\left(\boldsymbol{R}^{n+1}\right)$.

Lemma 3.3. If $|\nu| \leqq 1$, then the functional $\mathscr{F}_{\varepsilon}$ is lower semicontinuous with respect to $L^{1}\left(S_{T}\right)$-convergence.

Proof. Let $E_{h} \rightarrow E$ in $L^{1}\left(S_{T}\right)$, and suppose there exists $\sigma>0$ such that the inequality

$$
\mathscr{F}_{\varepsilon}(E)>\mathscr{F}_{\varepsilon}\left(E_{h}\right)+\sigma
$$

or equivalently

$$
\begin{aligned}
\int_{S_{T}}\left|D \varphi_{E_{h}}\right|< & \int_{S_{T}}\left|D \varphi_{E}\right|+\nu \int_{t=0}\left(\varphi_{E}-\varphi_{E_{h}}\right) d H_{n} \\
& +\int_{t=T}\left(\varphi_{E}-\varphi_{E_{h}}\right) d H_{n}+\varepsilon \int_{S_{T}} t\left(\varphi_{E}-\varphi_{E_{h}}\right) d z-\sigma
\end{aligned}
$$

holds, for infinite indices $h$. Combining (3.7) with the estimate

$$
\int_{\partial S_{T}}|f| d H_{n} \leqq \int_{S_{T}(\delta)}|D f|+c(\delta) \int_{S_{T}}|f| d z
$$

(which holds for every $f \in B V\left(S_{T}\right)$ and $\delta \in(0,-T / 2)$, provided we define

$$
S_{T}(\delta)=\left\{z \in S_{T}: \operatorname{dist}\left(z, \partial S_{T}\right)<\delta\right\},
$$

see [9]), we then obtain

$$
\begin{aligned}
\int_{S_{T}-\bar{S}_{T}(\delta)}\left|D \varphi_{E_{h}}\right|< & \int_{S_{T}}\left|D \varphi_{E}\right|+\int_{S_{T^{(\delta)}}}\left|D \varphi_{E}\right| \\
& +(c(\delta)-\varepsilon T) \int_{S_{T}}\left|\varphi_{E}-\varphi_{E_{h}}\right| d z-\sigma
\end{aligned}
$$

from which we derive a contradiction, letting $h \rightarrow+\infty$ and then $\delta \downarrow 0$, and taking into account the lower semicontinuity of perimeter functional (see [5]).

According to the previous lemmas, we can state the following existence result:

THEOREM 3.4. For every $T<0, V>0,-1<\nu \leqq 1$ and for every 
$\varepsilon>0$, the functional $\mathscr{F}_{\varepsilon}$ defined by (3.1) has a minimum $E_{\varepsilon}$ in the class $\mathscr{E}_{T}$, which is a rotationally symmetric set, so that we can write:

$$
E_{\varepsilon}=\left\{(x, t) \in S_{T}:|x|<\rho_{\varepsilon}(t)\right\}
$$

for a suitable function $\rho_{\varepsilon} \in B V((T, 0))$.

4. Some properties of $\varepsilon$-solutions. We study in this section the behaviour of the solutions $E_{\varepsilon}$, found in Theorem 3.4 , as $\varepsilon$ tends to zero.

From now on, we shall assume that $T=T(\nu, V)$ is a negative number, smaller than the minimum height $Q_{0}(\nu, V)$ of the solution $E_{0}$ in the absence of gravity (see (2.4); the data $\nu$ and $V$ will be held fixed). Moreover, we shall write $S$ instead of $S_{T}$.

Proposition 4.1. If $|\nu|<1$, then

$$
\begin{gathered}
E_{\varepsilon} \longrightarrow E_{0} \text { in } L^{1}(S) \\
\mathscr{F}_{\varepsilon}\left(E_{\varepsilon}\right) \longrightarrow \mathscr{F}_{0}\left(E_{0}\right)
\end{gathered}
$$

$a s \varepsilon \rightarrow 0$.

Proof. Let $A \subset S$ be a fixed set of volume $V$. We have for every $\varepsilon>0$

$$
\mathscr{F}_{\varepsilon}\left(E_{\mathrm{\varepsilon}}\right) \leqq \mathscr{F}_{\varepsilon}(A)<\text { const. },
$$

so that (3.4) implies that the perimeters of the $E_{\varepsilon}$ 's are uniformly bounded.

Now, the sets $E_{\varepsilon}$ are all included in a fixed cylinder, as we have pointed out during the proof of Lemma 3.2. This fact, together with the compactness result already mentioned, entails the convergence in $L^{1}\left(\boldsymbol{R}^{n+1}\right)$ of the $E_{\varepsilon}$ 's to some set $F \in \mathscr{E}_{T}$. We claim that $F$ minimizes functional $\mathscr{F}_{0}$ defined by (2.1). Indeed, by the assumption $T<Q_{0}$ we have $E_{0} \in \mathscr{E}_{T}$, and moreover we have

$$
\mathscr{F}_{0}\left(E_{\varepsilon}\right) \leqq \mathscr{F}_{0}\left(E_{0}\right)-\varepsilon \int_{S} t\left(\varphi_{E_{\varepsilon}}(z)-\varphi_{E_{0}}(z)\right) d z
$$

owing to the minimality of $E_{\varepsilon}$ 's.

Letting $\varepsilon \rightarrow 0$ in (4.3), we obtain from the lower semicontinuity of $\mathscr{F}_{0}$ with respect to $L^{1}$-convergence

$$
\mathscr{F}_{0}(F) \leqq \mathscr{F}_{0}\left(E_{0}\right) \text {. }
$$

Uniqueness of the solution $E_{0}$ allows to conclude that $F=E_{0}$, thus 
proving (4.1).

From (4.3) we derive also

$$
\mathscr{F}_{0}\left(E_{0}\right) \leqq \liminf _{\varepsilon \rightarrow 0} \mathscr{F}_{\varepsilon}\left(E_{\varepsilon}\right) \leqq \limsup _{\varepsilon \rightarrow 0} \mathscr{F}_{\varepsilon}\left(E_{\varepsilon}\right) \leqq \mathscr{F}_{0}\left(E_{0}\right)
$$

which yields (4.2).

Lemma 4.2. There holds for $|\nu|<1$ :

$$
\lim _{\varepsilon \rightarrow 0} \int_{t=0} \varphi_{E_{\varepsilon}} d H_{n}=\int_{t=0} \varphi_{E_{0}} d H_{n} .
$$

Proof. Let $\varepsilon_{h} \rightarrow 0$ be such that, for the corresponding solutions $E_{h} \equiv E_{\varepsilon_{h}}$, there exists

$$
\lim _{h \rightarrow+\infty} \int_{t=0} \varphi_{E_{h}} d H_{n}=a .
$$

Denote by $B_{h}, h=0,1,2, \cdots$, the intersection of the half-space $\{t<0\}$ with a suitable ball in $\boldsymbol{R}^{n+1}$, centered on the $t$-axis and chosen in such a way that

$$
\begin{array}{ll}
\left|B_{h}\right|=V & \forall h \\
\int_{t=0} \varphi_{B_{h}} d H_{n}=\int_{t=0} \varphi_{E_{h}} d H_{n} & \forall h \geqq 1 \\
\int_{t=0} \varphi_{B_{0}} d H_{n}=a . &
\end{array}
$$

In view of the isoperimetric inequality ([8], [18]) we have, for every $h \geqq 1$ :

$$
\int_{t<0}\left|D \mathcal{P}_{E_{h}}\right| \geqq \int_{t<0}\left|D \mathcal{P}_{B_{h}}\right|
$$

and hence

$$
\mathscr{F}_{\varepsilon_{h}}\left(E_{h}\right) \geqq \int_{t<0}\left|D \varphi_{B_{h}}\right|+\nu \int_{t=0} \varphi_{B_{h}} d H_{n}+\varepsilon_{h} \int_{S} t \varphi_{E_{h}}(z) d z
$$

letting $h \rightarrow+\infty$ and recalling (4.2) we obtain

$$
\mathscr{F}_{0}\left(E_{0}\right) \geqq \mathscr{F}_{0}\left(B_{0}\right) \text {. }
$$

Now, $E_{0}$ is the unique minimum of $\mathscr{F}_{0}$, so that $E_{0}=B_{0}$ and

$$
\lim _{h \rightarrow+\infty} \int_{t=0} \mathcal{P}_{E_{h}} d H_{n}=\int_{t=0} \varphi_{E_{0}} d H_{n}
$$

from which (4.4) follows at once. 
Lemma 4.3. There holds for $|\nu|<1$

$$
\begin{gathered}
\lim _{\varepsilon \rightarrow 0} \int_{S}\left|D \varphi_{E_{\varepsilon}}\right|=\int_{S}\left|D \varphi_{E_{0}}\right| \\
\lim _{\varepsilon \rightarrow 0} \int_{t=T} \varphi_{E_{\varepsilon}} d H_{n}=0 .
\end{gathered}
$$

Proof. From (4.3) we have

$$
\int_{S}\left|D \varphi_{E_{\varepsilon}}\right|+\nu \int_{t=0} \varphi_{E_{\varepsilon}} d H_{n}+\varepsilon \int_{S} t \varphi_{E_{\varepsilon}}(z) d z \leqq \mathscr{F}_{\varepsilon}\left(E_{\varepsilon}\right) \leqq \mathscr{F}_{0}\left(E_{0}\right)
$$

and then, from (4.4)

$$
\limsup _{\varepsilon \rightarrow 0} \int_{S}\left|D \varphi_{E_{\varepsilon}}\right| \leqq \int_{S}\left|D \varphi_{E_{0}}\right|
$$

while

$$
\int_{S}\left|D \varphi_{E_{0}}\right| \leqq \liminf _{\varepsilon \rightarrow 0} \int_{S}\left|D \varphi_{E_{\varepsilon}}\right|
$$

follows from (4.1) and lower semicontinuity of the perimeter functional.

REMARK 4.4. An obvious consequence of the previous results is the (pointwise a.e.) convergence of the functions defining the rotationally symmetric solutions $E_{\mathrm{s}}, E_{0}$. We recall (see Theorem 3.4) that such functions $\rho_{\varepsilon}:[T, 0] \rightarrow[0,+\infty)$ are defined through the relations

$$
E_{\varepsilon}=\left\{(x, t) \in S:|x|<\rho_{\varepsilon}(t)\right\}, \quad \varepsilon \geqq 0 .
$$

Since modifications of any set of finite perimeter by sets of zeromeasure do not affect its perimeter, we may assume the existence of both the one-sided limits of $\rho_{\varepsilon}$, at every point in the interval $(T, 0)$. Such limits do coincide, except for a countable set of points.

We have actually the following stronger result:

THEOREM 4.5. The convergence $\rho_{\varepsilon} \rightarrow \rho_{0}$ is uniform on $(T, 0)$.

Proof. We shall prove the statement in the theorem by showing that every subsequence of $\left\{\rho_{\varepsilon}\right\}_{\varepsilon>0}$ admits a subsequence uniformly convergent to $\rho_{0}$ on $(T, 0)$.

Let $\varepsilon_{h} \rightarrow 0$; for the corresponding sequence $\rho_{h}=\rho_{\varepsilon_{h}}$ we have, in view of (4.1)

$$
\lim _{h \rightarrow+\infty} \omega_{n} \int_{T}^{0}\left|\rho_{h}^{n}(t)-\rho_{0}^{n}(t)\right| d t=0
$$


Hence, a subsequence (not relabeled) of $\left\{\rho_{h}\right\}$ satisfies, for almost all $t \in[T, 0]:$

$$
\lim _{h \rightarrow+\infty} \rho_{h}(t)=\rho_{0}(t) .
$$

Assume by contradiction there exist $\sigma>0$ and a monotone sequence $\left\{t_{h}\right\} \subset[T, 0]$ such that

$$
\left\{\begin{array}{l}
\left|\rho_{h}\left(t_{h}\right)-\rho_{0}\left(t_{h}\right)\right|>\sigma \\
\lim _{h \rightarrow+\infty} t_{h}=t_{0} .
\end{array}\right.
$$

If $\left\{t_{h}\right\}$ is not decreasing, then choose $\bar{t} \in(T, 0)$ such that

$$
\begin{gathered}
\bar{t}<t_{0} \\
\lim _{h \rightarrow+\infty} \rho_{h}(\bar{t})=\rho_{0}(\bar{t})
\end{gathered}
$$

(in the opposite case, i.e., $\left\{t_{h}\right\}$ not increasing, choose $\bar{t}$ s.t. (4.11) holds with $\bar{t}>t_{0}$ ).

There follows that, for sufficiently great $h$

$$
\begin{aligned}
\int_{S}\left|D \varphi_{E_{h}}\right| & =\int_{t_{h}<t<0}\left|D \varphi_{E_{h}}\right|+\int_{\bar{t} \leqq t \leqq t_{h}}\left|D \varphi_{\bar{t}_{h}}\right|+\int_{T<t<\bar{t}}\left|D \varphi_{E_{h}}\right| \\
& \geqq \int_{t_{0}<t<0}\left|D \varphi_{E_{h}}\right|+\omega_{n}\left|\rho_{h}^{n}\left(t_{h}\right)-\rho_{h}^{n}(\bar{t})\right|+\int_{T<t<\bar{t}}\left|D \varphi_{E_{h}}\right| .
\end{aligned}
$$

On the other hand, form (4.9), (4.11) and from the continuity of $\rho_{0}$, we derive

$$
\left|\rho_{h}\left(t_{h}\right)-\rho_{h}(\bar{t})\right| \geqq \sigma-\left(\left|\rho_{0}\left(t_{h}\right)-\rho_{0}(\bar{t})\right|+\left|\rho_{0}(\bar{t})-\rho_{h}(\bar{t})\right|\right) \geqq \frac{\sigma}{2}
$$

provided that $\bar{t}$ is close to $t_{0}$ and $h$ is great. Therefore,

$$
\int_{S}\left|D \varphi_{E_{h}}\right| \geqq \int_{t_{0}<t<0}\left|D \varphi_{E_{h}}\right|+\omega_{n}\left(\frac{\sigma}{2}\right)^{n}+\int_{T<t<\bar{t}}\left|D \varphi_{E_{h}}\right|
$$

and letting $h \rightarrow+\infty$ we obtain

$$
\liminf _{h \rightarrow \infty} \int_{S}\left|D \varphi_{E_{h}}\right| \geqq \int_{t_{0}<t<0}\left|D \varphi_{E_{0}}\right|+\omega_{n}\left(\frac{\sigma}{2}\right)^{n}+\int_{T<t<\bar{t}}\left|D \varphi_{E_{0}}\right|
$$

which contrasts with (4.6) as $\bar{t}$ approaches $t_{0}$.

5. Regularity of $\varepsilon$-solutions. The main result in this section is the following.

TheOREM 5.1. Let $t_{0} \in(T, 0)$. If 


$$
\liminf _{t \rightarrow t_{0}} \rho_{\varepsilon}(t)>0
$$

then there exists a neighborhood $U$ of $t_{0}$ such that $\rho_{\varepsilon} \in C^{2}(U)$.

Proof. In view of Remark 4.4 we can find a neighborhood $W$ of $t_{0}$, such that

$$
\inf _{W} \rho_{\varepsilon}(t)=m_{\varepsilon}>0 .
$$

Hence, there exists a ball $B$ lying in the plane $\left\{x_{1}=0\right\}$ and centered at $\left(0, \cdots, t_{0}\right)$, which satisfies

$$
\begin{aligned}
E_{\varepsilon} \cap\left(\boldsymbol{R}^{+} \times B\right)= & \left\{\left(x_{1}, y, t\right) \in \boldsymbol{R}^{n+1}:(y, t) \in B,\right. \\
& \left.0<x_{1}<f_{\varepsilon}(y, t)=\left(\rho_{\varepsilon}^{2}(t)-|y|^{2}\right)^{1 / 2}\right\}
\end{aligned}
$$

where $y=\left(x_{2}, \cdots, x_{n}\right) \in \boldsymbol{R}^{n-1}$ and $f_{\varepsilon}(y, t) \geqq \psi_{\varepsilon}(y, t) \equiv m_{\varepsilon} / 2$. Function $f_{\varepsilon}$ belongs to $B V(B)$ and, owing to the minimum property of $\rho_{\varepsilon}$, it minimizes the functional

$$
I_{\varepsilon}(u)=\int_{B} \sqrt{1+|D u|^{2}}+\int_{\partial B}\left|u-f_{\varepsilon}\right| d H_{n-1}+\varepsilon \int_{B} t u d y d t
$$

in the function class

$$
H_{\varepsilon}=\left\{u \in B V(B): u \geqq \psi_{\varepsilon}, \int_{B}\left(u-\psi_{\varepsilon}\right) d y d t=\int_{B}\left(f_{\varepsilon}-\psi_{\varepsilon}\right) d y d t\right\} .
$$

We then conclude that $f_{\varepsilon}$ minimizes the functional

$$
I_{\varepsilon}(u)+\lambda_{\varepsilon} \int_{B} u d y d t
$$

in the function class

$$
K_{\varepsilon}=\left\{u \in B V(B): u \geqq \psi_{\varepsilon}\right\}
$$

as well, for a suitable multiplier $\lambda_{\varepsilon} \in \boldsymbol{R}$, whose existence is granted by the results in the Appendix. From known regularity results (see e.g., [10]) we obtain $f_{\varepsilon} \in C^{2, \alpha}(B), 0 \leqq \alpha<1$, thus proving the theorem.

REMARK 5.2. As a consequence of the theorem we can state, that if $\lim \inf _{t \rightarrow t_{0}} \rho_{\varepsilon}(t)>0 \forall t_{0} \in(a, b) \subset(T, 0)$, then $\rho_{\varepsilon} \in C^{2}(a, b)$ and there exists a real number $\Lambda_{s}$ such that

$$
\left(\frac{\rho_{\varepsilon}^{\prime}}{\sqrt{1+\rho_{\varepsilon}^{\prime 2}}}\right)^{\prime}-\frac{n-1}{\rho_{\varepsilon} \sqrt{1+\rho_{\varepsilon}^{\prime 2}}}=\varepsilon t+\Lambda_{\varepsilon} \quad \forall t \in(a, b) .
$$

Now, let $[a, b]$ be a sub-interval of $\left(Q_{0}, 0\right)$ (we recall that $Q_{0}$ denotes 
the height of the apex of the drop below the contact plane, in the absence of gravity; see (2.4)). In view of Theorem 4.5 we conclude, that for small $\varepsilon$ each function $\rho_{\varepsilon}$ is greater then a positive constant, independent of $\varepsilon$, in the whole interval $(a, b)$. This fact allows the proof of the following result, concerning the convergence of the $\Lambda_{\varepsilon}$ 's defined in the preceding remark.

\section{Proposition 5.3. There holds}

$$
\lim _{s \rightarrow 0} \Lambda_{c}=-\frac{n}{R_{0}}=\Lambda_{0}
$$

with $R_{0}$ defined by (2.2), (2.3).

Proof. As in the proof of Theorem 5.1, we can find a ball $B \subset$ $\left\{x_{1}=0\right\}$ (with radius independent of $\varepsilon$ ) and a function $\psi$ positive on $B$, such that

$$
\left\{\begin{array}{l}
B \cap\{y=0\} \subset(a, b) \\
f_{c} \geqq 2 \text { is on } \quad B
\end{array}\right.
$$

and $f$ minimizes the functional

$$
\int_{B} \sqrt{1+|D u|^{2}}+\int_{\partial B}\left|u-f_{\varepsilon}\right| d H_{n-1}+\varepsilon \int_{B} t u d y d t+\lambda_{\varepsilon} \int_{B} u d y d t
$$

in the class $\{u \in B V(B): u \geqq \psi\}$.

About the multipliers $\lambda_{s}$ we know the estimate (see (A6), (A7) in the Appendix):

$$
C_{2}(B) \leqq \lambda_{s} \leqq \frac{C_{1}(\gamma, B)+\int_{\partial B}\left|f_{\varepsilon}\right| d H_{n-1}}{V_{\varepsilon}}+c(B)
$$

where

$$
V_{\varepsilon}=\int_{B}\left(f_{\varepsilon}-\dot{s}\right) d y d t
$$

We may then assume $\lambda_{\varepsilon} \rightarrow \lambda_{0}$, from which we conclude that the function $f_{0}$, limit of the $f_{s}^{\prime}$ 's, minimizes functional (5.6) with $\varepsilon=0$. From (5.3) we have therefore:

$$
\lambda_{0}=-\frac{n}{R_{0}}, \quad \lambda_{\varepsilon}=\Lambda_{\varepsilon} .
$$

6. The existence theorem. So far we obtained a minimum $E_{\varepsilon}$ of the functional $\mathscr{F}_{\varepsilon}$ defined by $(3.1)$, for every $\varepsilon>0$. In order to 
get the existence of a pendent drop it suffices to show, that when $\varepsilon$ is small enough, there exists $t_{\varepsilon} \in(T, 0)$ such that $\lim _{i n f} \operatorname{int}_{t \rightarrow} \rho_{\varepsilon}(t)=0$.

Indeed, in this situation the set

$$
G_{\varepsilon}=E_{\varepsilon} \cap\left\{t>t_{\varepsilon}\right\}
$$

clearly yields the minimum value for $\mathscr{F}_{\varepsilon}$, when compared with the subsets of the strip $S$ having finite perimeter and volume equal to $G_{s} \mid$. In other words, following the definition given in $\S 1$, we can assert that $G_{\varepsilon}$ is a local minimum of functional (0.1), with $\kappa=\varepsilon$.

To this end, we compare the boundaries of solutions $E_{\varepsilon}$ with surfaces of constant mean curvature (see [4]).

We begin by noting that if $\rho_{\varepsilon}^{\prime}(\bar{t})>0$, then there exists, in a suitable neighborhood $U$ of the point $\bar{r}=\rho_{c}(\bar{t})$, the inverse function $u_{\varepsilon}(r)$ of $\rho_{\varepsilon}$; moreover, from (5.3) we deduce

$$
\frac{1}{r^{n-1}}\left(\frac{r^{n-1} u_{\varepsilon}^{\prime}}{\sqrt{1+u_{c}^{\prime 2}}}\right)^{\prime}=-\varepsilon u_{\varepsilon}-\Lambda_{\varepsilon} \quad(r \in U) .
$$

On the contrary, if $\rho_{s}^{\prime}(\bar{t})<0$, then the following equation holds instead of (6.1)

$$
\frac{1}{r^{n-1}}\left(\frac{r^{n-1} u_{\varepsilon}^{\prime}}{\sqrt{1+u_{\varepsilon}^{\prime 2}}}\right)^{\prime}=\varepsilon u_{\varepsilon}+A_{\varepsilon} \quad(r \in U) .
$$

In either case, denoting by $\psi_{u_{\varepsilon}}(r)$ the angle between the tangent line to $u_{\varepsilon}$ and the $r$-axis, measured counterclockwise from the $r$-axis itself, we can combine (6.1) and (6.2) in the single equation

$$
\frac{1}{r^{n-1}}\left(r^{n-1} \sin \psi_{u_{s}}(r)\right)^{\prime}=-\varepsilon u_{\varepsilon}(r)-\Lambda_{\varepsilon} .
$$

We also need the following results (see [4]):

Lemma 6.1. Let $u(r), v(r)$ be functions defined over $0 \leqq a \leqq r \leqq b$, s.t.

$$
\left(r^{n-1} \sin \psi_{v}(r)\right)^{\prime} \geqq\left(r^{n-1} \sin \psi_{u}(r)\right)^{\prime} .
$$

If $\sin \psi_{v}(a) \geqq \sin \psi_{u}(a)$, then $\sin \psi_{v}(b) \geqq \sin \psi_{u}(b)$, and equality holds if and only if $u=v+$ const in $[a, b]$.

Lemma 6.2. For every $H>0$ and for every a such that

$$
0<a<\frac{n-1}{n H}
$$

there exists an unique $b$ with 


$$
\frac{n-1}{n H}<b<\frac{1}{H}
$$

and an increasing function $v:[a, b] \rightarrow \boldsymbol{R}$ for which

$$
\begin{gathered}
\frac{1}{r^{n-1}}\left(r^{n-1} \sin \psi_{v}(r)\right)^{\prime}=n H \quad(r \in(a, b)) \\
\sin \psi_{v}(a)=\sin \psi_{v}(b)=1 .
\end{gathered}
$$

Moreover, there holds

$$
v(b)-v(a) \leqq \frac{c(n)}{H} .
$$

The proof of Lemma 6.1 is quite obvious, so we outline only that of Lemma 6.2. We point out that the surface obtained by rotating the graph of function $v(r)$ in the lemma (which is part of an ellipse roulade when $n=2$, see [4]) about the vertical axis, has constant mean curvature $H$.

Integrating (6.6) yields

$$
v^{\prime}(r)=\frac{g(r)}{\sqrt{1-g^{2}(r)}}
$$

where $g(r)=H r+B r^{1-n}$.

For the constant of integration $B$ we have, in view of (6.7)

$$
B=a^{n-1}-H a^{n}=b^{n-1}-H b^{n}>0
$$

and letting $c=(n-1) / n H$ we get from (6.9)

$$
v(b)-v(a) \leqq \frac{1}{\sqrt{2}}\left(\int_{a}^{c} \frac{d r}{\sqrt{1-g(r)}}+\int_{c}^{b} \frac{d r}{\sqrt{1-g(r)}}\right) .
$$

Defining

$$
\begin{aligned}
& \alpha(r)=(r-a)(1-g(r))^{-1} \\
& \beta(r)=(b-r)(1-g(r))^{-1}
\end{aligned}
$$

and noting that $\alpha^{\prime}(r)>0>\beta^{\prime}(r)$, we have from (6.10)

$$
v(b)-v(a) \leqq \sqrt{2}(\sqrt{\alpha(c)} \sqrt{c-a}+\sqrt{\beta(c)} \sqrt{b-c}) .
$$

Now, functions

$$
\begin{array}{ll}
\sqrt{\alpha(c)(c-a)}=(c-a)(1-g(c))^{-1 / 2} & 0<a<c \\
\sqrt{\beta(c)(b-c)}=(b-c)(1-g(c))^{-1 / 2} & c<b<\frac{1}{H}
\end{array}
$$

are decreasing and we can conclude

$$
v(b)-v(a) \leqq \frac{c(n)}{H} .
$$


We are now in position to prove the following

THEOREM 6.3. If $T<Q_{0}-3 c(n) R_{0}$ and $0<\varepsilon<\varepsilon_{0}(T)$, then there exists $t_{\varepsilon} \in(T, 0)$ such that

$$
\liminf _{t \rightarrow t_{\varepsilon}} \rho_{\varepsilon}(t)=0 .
$$

Proof. Assume by contradiction the existence of a sequence $\left\{\varepsilon_{h}\right\}$ converging to zero, such that

$$
\liminf _{s \rightarrow t} \rho_{h}(s)>0
$$

for every $t \in(T, 0)$ and every $h$, with $\rho_{h} \equiv \rho_{\varepsilon_{h}}$.

In view of Remark 5.2, for every $h$ we have $\rho_{h} \in C^{2}(T, 0)$; moreover $\rho_{h}$ satisfies the Euler equation

$$
\left(\frac{\rho_{h}^{\prime}}{\sqrt{1+\rho_{h}^{\prime 2}}}\right)^{\prime}-\frac{n-1}{\rho_{h} \sqrt{1+\rho_{h}^{\prime 2}}}=\varepsilon_{h} t+\Lambda_{h}
$$

in which

$$
\lim _{h \uparrow+\infty} \Lambda_{h}=-\frac{n}{R_{0}}
$$

(see (5.4)). Now, let $T_{1}=T+\left(Q_{0}-T\right) / 3$ and $T_{2}=T+\left(2\left(Q_{0}-T\right)\right) / 3$, and denote by $t_{h} \in\left(T_{1}, T_{2}\right)$ a point such that $\rho_{h}^{\prime}\left(t_{h}\right) \neq 0$. For the sake of definiteness, assume $\rho_{h}^{\prime}\left(t_{h}\right)>0$, since in the opposite case one can proceed analogously.

Let $a_{h}=\rho_{h}\left(t_{h}\right)$ and $H_{h}=\left(-\varepsilon_{h} T-\Lambda_{h}\right) / n$; there follows from Theorem 4.5 and (6.12) that

$$
\lim _{h \rightarrow+\infty} a_{h}=0 ; \lim _{h \rightarrow+\infty} H_{h}=\frac{1}{R_{0}},
$$

so that, in view of Lemma 6.2, we can find (for sufficiently great $h$ ) $b_{h} \in\left((n-1) / n H_{h}, 1 / H_{h}\right)$ and $v_{h}:\left[a_{h}, b_{h}\right] \rightarrow \boldsymbol{R}$ satisfying (6.6), (6.7) together with $v_{h}\left(a_{h}\right)=t_{h}$.

An application of Lemma 6.1 to $u_{h}, v_{h}$ ( $u_{h}$ denotes the inverse function of $\rho_{h}$ ) allows the conclusion that $u_{h}$ is defined over the whole interval $\left[a_{h}, b_{h}\right]$, and verifies there

$$
u_{h}(r) \leqq v_{h}(r) \quad \forall r \in\left[a_{h}, b_{h}\right] .
$$

Finally, as a consequence of the choice of $T$, we have from (6.8)

$$
v_{h}\left(b_{h}\right) \leqq \frac{c(n)}{H_{h}}+T_{2}<Q_{0}+c(n)\left(\frac{1}{H_{h}}-R_{0}\right)
$$

and therefore, for great $h$ 


$$
v_{h}\left(b_{h}\right)<Q_{0} .
$$

Now, in the interval $\left(T, Q_{0}\right)$ the sequence $\left\{\rho_{h}\right\}$ tends uniformly to zero (Theorem 4.5), so contradicting the fact that

$$
\rho_{h}\left(u_{h}\left(b_{h}\right)\right)=b_{h}>\frac{n-1}{n H_{h}} \longrightarrow \frac{n-1}{n} R_{0}>0 .
$$

From the minimality of $E_{\varepsilon}$ there follows that the set of points $t_{\varepsilon} \in(T, 0)$ such that $\liminf _{t \rightarrow t_{\varepsilon}} \rho_{\varepsilon}(t)=0$ is a closed interval in $(T, 0)$ (of course, it may consist of a single point). Denoting by $Q_{\varepsilon}$ the maximum of such interval, we conclude that $\rho_{\varepsilon}$ is positive and regular in $\left(Q_{\varepsilon}, 0\right) ; Q_{\varepsilon}$ thus represents the minimum height of the pendent drop

$$
G_{\varepsilon}=\left\{(x, t): t \in\left(Q_{\varepsilon}, 0\right),|x|<\rho_{\varepsilon}(t)\right\} .
$$

7. Regularity at the vertex. At this point we have proved the existence of a local minimum of (0.1) for sufficiently small $\kappa$; nevertheless, we cannot say anything about the effective volume of such solution, nor about the effective smallness of $\kappa$.

It is however clear, that by a homothetic transformation of the co-ordinate system we can expand (or contract) our solution so that it becomes a local minimum of (0.1) (for a different $\kappa$, of course) among sets of prescribed volume $V$; or, so that it becomes a local minimum of (0.1) for a prescribed value of $\kappa>0$, in the class of sets having its own volume (which remains unspecified).

Thus, we can obtain by this method pendent drops of fixed volume in a weak gravitational field, or pendent drops of small volume in a prescribed gravitational field.

The following considerations are devoted to the study of the behavior of our solution near its minimum height $Q_{c}$.

Let $\tau \in\left(Q_{\varepsilon}, 0\right)$, and denote by $B(\tau)$ the ball centered on the $t$-axis, passing through the boundary point $\left(\rho_{\varepsilon}(\tau), 0, \cdots 0, \tau\right) \in \boldsymbol{R}^{n+1}$ and satisfying (see (6.14)):

$$
H_{n+1}\left[\left(G_{\varepsilon}-B(\tau)\right) \cap\left\{Q_{\varepsilon}<t<\tau\right\}\right]=H_{n+1}\left[B(\tau) \cap\left\{t<Q_{\varepsilon}\right\}\right]
$$

$R(\tau)$ will denote the radius of $B(\tau)$.

Lemma 7.1. If

$$
\lim _{t \downarrow Q_{\varepsilon}} \rho_{s}(t)=L>0
$$

then there exists $\tau \in\left(Q_{r}, 0\right)$ such that

$$
B(\tau) \cap\left\{Q_{\varepsilon}<t<\tau\right\} \subset G_{\varepsilon} \cap\left\{Q_{s}<t<\tau\right\} .
$$

Proof. It follows from (7.2) that 


$$
\lim _{\tau \downarrow Q_{\varepsilon}} R(\tau)=+\infty .
$$

If the assertion in the lemma were false, then we could find, in view of (7.4) and the mean value theorem, a sequence $\left\{t_{j}\right\}$ converging to $Q_{\varepsilon}$ from above, such that

$$
\lim _{j \rightarrow+\infty} \rho_{\varepsilon}^{\prime}\left(t_{j}\right)=+\infty .
$$

Taking into account (7.2), (7.5), we derive from (5.3)

$$
\frac{\rho_{\varepsilon}^{\prime \prime}\left(t_{j}\right)}{\sqrt{\left(1+\rho_{s}^{\prime 2}\left(t_{j}\right)\right)^{3}}}=\frac{n-1}{\rho_{\varepsilon}\left(t_{j}\right) \sqrt{1+\rho_{s}^{\prime 2}\left(t_{j}\right)}}+\varepsilon t_{j}+\Lambda_{\varepsilon}<0
$$

when $j>j_{0}$, since, as we shall see in the following remark, there holds $\varepsilon Q_{\varepsilon}+A_{\varepsilon}<0$. Then we can easily conclude that $\rho_{\varepsilon}^{\prime \prime}(t)<0 \forall t \in$ $\left(Q_{s}, t_{\partial_{0}}\right)$. Hence, we can describe the boundary of $G_{\varepsilon}$, lying in a neighborhood of the point $\left(L, 0, \cdots 0, Q_{\varepsilon}\right) \in \boldsymbol{R}^{n+1}$, as the graph of a suitable function $t=u(x)$ which, in view of the results in $\S 5$, would be analytic over its domain of definition; but this leads to a contradiction, since the (not-identically constant) function $u$ should be constant $\left(\equiv Q_{s}\right)$ over an open set.

COROLLARY 7.2. There holds

$$
\lim _{t \downarrow Q_{s}} \rho_{s}(t)=0 .
$$

Proof. If not (see Remark 4.4), we get from the lemma the existence of a ball $B(\tau)$ satisfying (7.3). Defining

$$
\widetilde{G}_{\varepsilon}= \begin{cases}G_{\varepsilon} & \text { for } t \geqq \tau \\ B(\tau) & \text { for } t \leqq \tau\end{cases}
$$

there follows from the isoperimetric inequality

$$
H_{n+1}\left(\widetilde{G}_{\varepsilon}\right)=H_{n+1}\left(G_{\varepsilon}\right) ; \mathscr{F}_{\varepsilon}\left(\widetilde{G}_{\varepsilon}\right)<\mathscr{F}_{\varepsilon}\left(G_{\varepsilon}\right),
$$

a contradiction.

REMARK 7.3. The inequality $\varepsilon Q_{\varepsilon}+A_{\varepsilon}<0$ we used in the proof of Lemma 7.1 really holds for a local minimum $G_{\varepsilon}$. Assuming the contrary, we derive from (5.3): $\rho_{\varepsilon}^{\prime \prime}(t)>0$ for every $t \in\left(Q_{\varepsilon}, 0\right)$, and then either $\partial G_{\varepsilon}$ is a (regular) graph in the $t$-direction, or there exists $\tau$ close to $Q_{\varepsilon}$ s.t. the corresponding ball $B(\tau)$ satisfies (7.3). In either case, as we saw just before, we are led to a contradiction.

CoRollary 7.4. There exists $\delta>0$ such that $\partial G_{\varepsilon} \cap B_{i}\left(0, \cdots, 0, Q_{\varepsilon}\right)$ is representable as a graph of an analytic function $t=u(x)$. 
Proof. Assume the contrary; then it would be possible to find a sequence $\left\{t_{j}\right\}$ of local maximum points for $\rho_{\varepsilon}$, decreasing to $Q_{\varepsilon}$. On the other hand, we have from (5.3)

$$
\rho_{\varepsilon}^{\prime \prime}\left(t_{j}\right) \geqq \frac{n-1}{\rho_{\varepsilon}\left(t_{j}\right)}+\varepsilon Q_{\varepsilon}+\Lambda_{\varepsilon}
$$

which is positive for $j$ great enough.

The assertion about analiticity follows from the methods of $\S 5$.

The preceding results, together with that of $\S 5$, can be summarized in the following

THEOREM 7.5. If $G$ is a local minimum of the functional $\mathscr{F}$ defined by (0.1), then $\partial G \cap\{t<0\}$ is an analytic n-dimensional manifold.

A. Appendix: Existence of multipliers. For convenience of the reader, we quote the proof of the following result, which we used in the proof of Theorem 5.1.

THEOREM A.1. If $f$ minimizes functional

$$
I(u)=\int_{B} \sqrt{1+|D u|^{2}}+\int_{\partial B}|u-f| d H_{n-1}+\int_{B} t u d y d t
$$

in the class

$$
H=\left\{u \in B V(B): u \geqq \psi, \int_{B}(u-\psi) d y d t=\int_{B}(f-\psi) d y d t=V>0\right\}
$$

( $\psi$ denotes a Lipschitz function over $B$ ), then there exists $\lambda \in \boldsymbol{R}$ such that $f$ minimizes $I(u)+\lambda \int_{B} u d y d t$ in the class $K=\{u \in B V(B): u \geqq \psi\}$.

The method of proof appears in [16], and involves various steps.

Lemma A.2. For any $\eta>0$ and $\lambda \in R$, the functional

$$
I_{\eta, \lambda}(u)=I(u)+\frac{\eta}{2} \int_{B} u^{2} d y d t+\lambda \int_{B} u d y d t
$$

attains its unique minimum $u_{\eta, \lambda} \in C^{0,1}(B)$ in the class $K$.

Proof. From the inequality $2 a b \leqq \sigma a^{2}+b^{2} / \sigma(\sigma>0)$ we derive

$$
I_{\eta, \lambda}(u) \geqq \int_{B} \sqrt{1+|D u|^{2}}+\int_{\partial B}|u-f| d H_{n-1}-\frac{1}{\eta}\left(\int_{B} t^{2} d y d t+\lambda^{2}|B|\right)
$$

that is, a lower bound for the functional and the necessary compactness property. 
Lower semicontinuity and strict convexity of $I_{\eta, \lambda}$ allow the conclusion about existence and uniqueness of the minimum in $K$. Its regularity can be obtained from a wellknown gradient estimate.

Lemma A.3. The function

$$
h(\lambda)=\int_{B}\left(u_{r, \lambda}-\psi\right) d y d t
$$

is continuous over $\boldsymbol{R}$ and satisfies

$$
\begin{gathered}
\lim _{\lambda \rightarrow+\infty} h(\lambda)=0 \\
\lim _{\lambda \rightarrow-\infty} h(\lambda)=+\infty .
\end{gathered}
$$

Proof. It is easy to show, that as $\lambda_{j}$ tends to $\lambda_{0}$, the sequence of corresponding minima $\left\{u_{\eta, \lambda_{j}}\right\}$ tends in $L^{1}(B)$ to a function $v$, which minimizes $I_{\eta, \lambda_{0}}$. Uniqueness implies therefore $v=u_{\eta, \lambda_{0}}$.

On the other hand, letting $c=\max _{B}\{-t\}$, we have

$$
\begin{aligned}
(\lambda-c) & \int_{B}\left(u_{\eta, \lambda}-\psi\right) d y d t \leqq I_{\eta, \lambda}\left(u_{\eta, \lambda}\right)-\int_{B} t u_{\eta, \lambda} d y d t \\
& -c \int_{B}\left(u_{\eta, \lambda}-\psi\right) d y d t-\lambda \int_{B} \psi d y d t \\
\leqq & \int_{B} \sqrt{1+|D \psi|^{2}}+\int_{\partial B}|\psi-f| d H_{n-1}+\frac{\eta}{2} \int_{B} \psi^{2} d y d t \\
& \quad-\int_{B}(t+c)\left(u_{\eta, \lambda}-\psi\right) d y d t \leqq c_{1}(\psi, B)+\int_{\partial B}|f| d H_{n-1}
\end{aligned}
$$

and this yields (A.1).

As far as (A.2) is concerned, define for $\delta \geqq 0$ and $\varphi \in H^{1,1}(B)$, $\varphi \geqq 0$ :

$$
\begin{aligned}
u_{\hat{o}} & =u_{\eta, \lambda}+\delta \varphi \\
\alpha(\delta) & =I_{\eta, \lambda}\left(u_{\tilde{\delta}}\right)-\int_{\hat{\partial} B}\left|u_{\hat{o}}-f\right| d H_{n-1}
\end{aligned}
$$

so that

$$
\alpha(0) \leqq \alpha(\delta)+\delta \int_{\partial B} \varphi d H_{n-1}
$$

Hence, the function $\alpha(\delta)+\delta \int_{\partial B} \varphi d H_{n-1}(\delta \geqq 0)$ attains its minimum at $\delta=0$, from which we obtain

$$
\begin{aligned}
& \int_{B} \frac{D u_{\eta, \lambda} \cdot D \varphi}{\sqrt{1+\left|D u_{\eta, \lambda}\right|^{2}}} d y d t+\eta \int_{B} u_{\eta, \lambda} \varphi d y d t \\
& \quad+\lambda \int_{B} \varphi d y d t+\int_{\partial B} \varphi d H_{n-1}+\int_{B} t \varphi d y d t \geqq 0 .
\end{aligned}
$$


In particular, choosing $\varphi \equiv 1$ we have

$$
\eta \int_{B} u_{\eta, \lambda} d y d t+\lambda|B| \geqq-H_{n-1}(\partial B)-\int_{B} t d y d t
$$

that is

$$
\begin{aligned}
& \eta \int_{B}\left(u_{\eta, \lambda}-\psi\right) d y d t+\lambda|B| \\
& \quad \geqq-H_{n-1}(\partial B)-\int_{B} t d y d t-\eta \int_{B} \psi d y d t \geqq c_{2}(\psi, B)
\end{aligned}
$$

and hence (A.2).

Proof of Theorem A.1. In view of Lemma A.3, for every $\eta>0$ there exists $\lambda_{\eta}$ s.t. $\int_{B}\left(u_{\eta, \lambda_{\eta}}-\psi\right) d y d t=V$. Moreover, from (A.3), (A.4) we have:

$$
\frac{c_{2}-\eta V}{|B|} \leqq \lambda_{r_{i}} \leqq \frac{c_{1}+\int_{\partial B}|f| d H_{n-1}}{V}+c
$$

hence we can assume $\lambda_{\eta} \rightarrow \lambda$ as $\eta \rightarrow 0$. There follows that $u_{\eta, \lambda_{\eta}}$ converges in $L^{1}(B)$ to a function $u_{0}$ minimizing $I_{0,2}$ in the class $K$.

From the relation

$$
\int_{B} u_{0} d y d t=\int_{B} f d y d t
$$

we derive $I_{0, \lambda}\left(u_{0}\right)=I_{0, \lambda}(f)$, which concludes the proof of the theorem.

REMARK A.4. From (A.5) we easily derive the following estimates concerning multipliers $\lambda$ :

$$
\begin{gathered}
\lambda \geqq C_{2}(B)=\frac{-H_{n-1}(\partial B)-\int_{B} t d y d t}{|B|} \\
\lambda \leqq \frac{C_{1}+\int_{\hat{\lambda} B}|f| d H_{n-1}}{V}+c \\
=\frac{\int_{B} \sqrt{1+|D \psi|^{2}}+\int_{\partial B}|\psi| d H_{n-1}+\int_{\partial B}|f| d H_{n-1}}{V}+\max _{B}\{-t\} .
\end{gathered}
$$

\section{REFERENCES}

1. G. Anzellotti, M. Giaquinta, U. Massari, G. Modica and L. Pepe, Note sul problema di Plateau, Editrice Tecnico Scientifica Pisa, (1974). 
2. P. Concus and R. Finn, On capillary free surfaces in a gravitational field, Acta Math., 132 (1974).

3. - A singular solution of the capillary equation I, II, Inv. Math., 29 (1975).

4. - The shape of a pendent liquid drop, Philos. Trans. Roy. Soc. London, Ser

A, 292 (1979).

5. E. De Giorgi, Su una teoria generale della misura $(r-1)$-dimensionale in uno spazio $a d r$ dimensioni, Ann. Mat. Pura e Appl., Serie IV, 36 (1954).

6 . Nuovi teoremi relativi alle misure $(r-1)$-dimensionali in uno spazio ad $r$ dimensioni, Ricerche di Mat., 4 (1955).

7. E. De Giorgi, F. Colombini and L. Piccinini, Frontiere orientate di misura minima e questioni collegate, Pisa, (1972).

8. E. De Giorgi, Sulla proprietà isoperimetrica della ipersfera, nella classe degli insiemi aventi frontiera orientata di misura finita, Memorie Acc. Naz. Lincei, Serie VIII, 5 (1958).

9. M. Emmer, Esistenza, unicità e regolarità delle superfici di equilibrio nei capillari, Ann. Univ. Ferrara, 18 (1973).

10. - Superfici di curvatura media assegnata con ostacolo, Ann, Mat. Pura e Appl., 109 (1976).

11. H. Federer, Geometric Measure Theory, Springer-Verlag, 1969.

12. R. Finn, Capillarity phenomena, Uspekhi Mat. Nauk., 29 (1974).

13. R. Finn and C. Gerhardt, The internal sphere condition and the capillarity problem, Ann. Mat. Pura e Appl. Serie IV, 112 (1977).

14. C. Gerhardt, Existence and regularity of capillary surfaces, Boll. U.M.I., 10 (1974). 15. Global regularity of the solutions to the capillarity problem, Ann. Scuola Norm. Sup. Pisa, Serie IV, 3 (1976).

16. On the capillarity problem with constant volume, Ann. Scuola Norm. Sup. Pisa, Serie IV, 2 (1975).

17. E. Giusti, Boundary value problems for non-parametric surfaces of prescribed mean curvature, Ann. Sc. Norm. Sup. Pisa, Serie IV, 3 (1976).

18. E. Gonzalez and G. Greco, Una nuova dimostrazione della proprietà isoperimetrica dell'ipersfera, Ann. Univ. Ferrara, 23 (1977).

19. E. Gonzalez, Introduccion a las fronteras minimas, Universidade de Sao Paulo, (1977). 20. - Sul problema della goccia appoggiata, Rend. Sem. Mat. Univ. Padova, 55 (1976).

21. - Regolarita per il problema della goccia appoggiata, Rend. Sem. Mat. Univ. Padova, 58 (1977).

22. E. Gonzalez and I. Tamanini, Convessità della goccia appoggiata, Rend. Sem. Mat. Univ. Padova, 58 (1977).

23. M. Miranda, Comportamento delle successioni convergenti di frontiere minimali, Rend. Sem. Mat. Univ. Padova, 38 (1967).

24. Distribuzioni aventi derivate misure e insiemi di perimetro localmente finito, Ann. Scuola Norm. Sup. Pisa, 18 (1964).

25. U. Massari and L. Pepe, Su una impostazione parametrica del problema dei capillari, Ann. Univ. Ferrara, 20 (1974).

26. - Su di una formulazione variazionale del problema dei capillari in assenza di gravità, Ann. Univ. Ferrara, 20 (1974).

27. L. Pepe, Analiticità delle superfici di equilıbrio dei capillari in ogni dimensione, Symposia Mathematica, 14 (1974).

28. E. Pitts, The stability of pendent liquid drops, Part 1. Drops formed in a narrow gap, J. Fluid Mech., 59 (1973).

29. - The stability of pendent liquid drops, Part 2. Axially symmetry, J. Fluid Mech., 63 (1974).

30. J. Spruck, On the existence of a capillary surface with prescribed contact angle, Comm. Pure Appl. Math., 28 (1975). 
31. L. M. Simon and J. Spruck, Existence and regularity of a capillary surface with prescribed contact angle, Arch. Rat. Mech. Analysis, 61 (1976).

32. I. Tamanini, Il problema della capillarità su domini non regolari, Rend. Sem. Mat. Univ. Padova, 56 (1977).

33. N. N. Ural'tseva, The solvability of the capillarity problem, Vestnik Leningrad Univ. $\mathrm{N}^{\circ} 19,4$ (1973).

34. H. C. Wente, An existence theorem for surfaces in equilibrium satisfying a volume constraint, Arch. Rat. Mech. Anal., 50 (1973).

Received May 11, 1979.

Dipartimento di Matematica

Libera Università degli Studi di Trento

38050-POVO (Trento), Italy 


\section{PACIFIC JOURNAL OF MATHEMATICS}

\section{EDITORS}

DONALD BABBITT (Managing Editor)

University of California

Los Angeles, CA 90024

Hugo Rossi

University of Utah

Salt Lake City, UT 84112

C. C. MOORE and ANDREW OGG

University of California

Berkeley, CA 94720
J. DUGUNDJI

Department of Mathematics

University of Southern California

Los Angeles, CA 90007

R. FinN and J. Milgram

Stanford University

Stanford, CA 94305

\section{ASSOCIATE EDITORS}
E. F. BECKENBACH
B. H. NeumanN
F. WOLF
K. YoSHIDA

\section{SUPPORTING INSTITUTIONS}

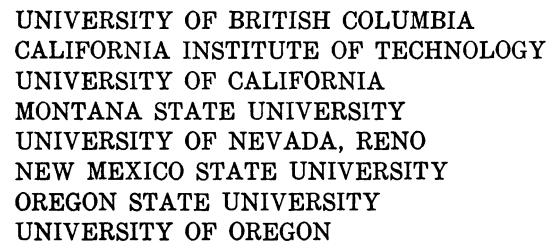

UNIVERSITY OF BRITISH COLUMBIA CALIFORNIA INSTITUTE OF TECHNOLOGY UNIVERSITY OF CALIFORNIA MONTANA STATE UNIVERSITY UNIVERSITY OF NEVADA, RENO NEW MEXICO STATE UNIVERSITY OREGON STATE UNIVERSITY UNIVERSITY OF OREGON

\author{
UNIVERSITY OF SOUTHERN CALIFORNIA \\ STANFORD UNIVERSITY \\ UNIVERSITY OF HAWAII \\ UNIVERSITY OF TOKYO \\ UNIVERSITY OF UTAH \\ WASHINGTON STATE UNIVERSITY \\ UNIVERSITY OF WASHINGTON
}

The Supporting Institutions listed above contribute to the cost of publication of this Journal, but they are not owners or publishers and have no responsibility for its content or policies.

Mathematical papers intended for publication in the Pacific Journal of Mathematics should be in typed form or offset-reproduced, (not dittoed), double spaced with large margins. Please do not use built up fractions in the text of the manuscript. However, you may use them in the displayed equations. Underline Greek letters in red, German in green, and script in blue. The first paragraph or two must be capable of being used separately as a synopsis of the entire paper. Please propose a heading for the odd numbered pages of less than 35 characters. Manuscripts, in triplicate, may be sent to any one of the editors. Please classify according to the scheme of Math. Reviews, Index to Vol. 39. Supply name and address of author to whom proofs should be sent. All other communications should be addressed to the managing editor, or Elaine Barth, University of California, Los Angeles, California, 90024.

50 reprints to each author are provided free for each article, only if page charges have been substantially paid. Additional copies may be obtained at cost in multiples of 50 .

The Pacific Journal of Mathematics is issued monthly as of January 1966. Regular subscription rate: $\$ 84.00$ a year (6 Vols., 12 issues). Special rato: $\$ 42.00$ a year to individual members of supporting institutions.

Subscriptions, orders for numbers issued in the last three calendar years, and changes of address shoud be sent to Pacific Journal of Mathematics, P.O. Box 969, Carmel Valley, CA 93924, U.S.A Old back numbers obtainable from Kraus Periodicals Co., Route 100, Millwood, NY 10546.

PUBLISHED BY PACIFIC JOURNAL OF MATHEMATICS, A NON-PROFIT CORPORATION

Printed at Kokusai Bunken Insatsusha (International Academic Printing Co., Ltd.). 8-8, 3-chome, Takadanobaba, Shinjuku-ku, Tokyo 160, Japan. 


\section{Pacific Journal of Mathematics \\ Vol. 88, No. $2 \quad$ April, 1980}

Reinhold Böhme, Stefan Hildebrandt and Engelbert Tausch, The two-dimensional analogue of the catenary ................. 247

Jean Ellen Taylor, Nonexistence of F-minimizing embedded disks ........ 279

Claus Gerhardt, A free boundary value problem for capillary surfaces ..... 285

Enrico Giusti, Generalized solutions for the mean curvature equation . . . . . 297

Jin-Tzu Chen, On the existence of capillary free surfaces in the absence of gravity.................................... 323

Leon M. Simon, Regularity of capillary surfaces over domains with corners.......................................... 363

Nicholas Jacob Korevaar, On the behavior of a capillary surface at a re-entrant corner.................................... 379

Henry Wente, The symmetry of sessile and pendent drops ............ 387

E. Gonzalez, Umberto Massari and I. Tamanini, Existence and regularity for the problem of a pendent liquid drop ...................... 399

Henry Wente, The stability of the axially symmetric pendent drop ........ 421

David Siegel, Height estimates for capillary surfaces . . . . . . . . . . . . 471

Bruce Edward Turkington, Height estimates for exterior problems of capillarity type ................................ 517

Robert Finn, The sessile liquid drop. I. Symmetric case .............. 541 